\title{
An Improved Control Strategy for the Bus Interlinking Converter Based on Three-level Topology in AC-DC Hybrid Microgrid
}

\author{
Yuan Ren ${ }^{1}$, Jinhao Wang ${ }^{1}$, Xiao Chang ${ }^{1}$, Yi Du ${ }^{2}$, Ying Zhang ${ }^{2}$, Yizhao Liu ${ }^{1}$, and Gangbin Wang ${ }^{1}$ \\ ${ }^{1}$ Electric Power Research Institute, State Grid Shanxi Electric Power Research Institute, Taiyuan 030000, China \\ ${ }^{2}$ College of Electrical and Power Engineering, Taiyuan University of Technology, Taiyuan 030000, China
}

\begin{abstract}
Considering the increasing requirements of the AC-DC hybrid microgrid (HMG) for the transmission power capacity and voltage level, the several bus interface converters (BICs) with the two-level PWM converter topology, need to be connected between AC bus and DC bus in parallel, which could generate circulating current and cycle power. The cost of the modular multilevel converter topology based BIC is too high. Hence, this paper proposes to apply the BIC with three-level topology to the HMG, and a droop control strategy with the midpoint potential hysteresis regulation link is designed, which can effectively suppress the fluctuation of midpoint potential without affecting the normal operation of the BIC in the grid connected or off mode. In addition, the principle of neutral point fluctuation and the process of hysteresis control are introduced, and the detailed design of dual-mode control strategy based on droop curve are also given. Finally, the effectiveness of the improved control strategy is verified by simulation.
\end{abstract}

\section{Introduction}

With the rapid development of the new energy power generation, research on the microgrid has been received more and more attention. According to the difference of bus voltages, the microgrid can be divided into DC microgrid, AC microgrid and AC-DC hybrid microgrid (HMG) [1]. Due to the AC-DC HMG has the advantages of receiving various $\mathrm{AC}$ sources and loads and DC sources and loads, saving a large number of AC-DC power converters and improving the economy and reliability of the AC-DC HMG, which has become the development trend of the microgrid [2-7].

Recently, the researches of AC-DC HMG mainly focus on the topology and operation mode of HMG [8] [9], the control strategies of the BIC [10], power quality optimization $[11,12]$ and coordinated control strategies of interlinking converters [13]. Moreover, the BICs as the connection hub of $\mathrm{AC}$ and $\mathrm{DC}$ sub networks, which need to control the bidirectional power between the $\mathrm{AC}$ and DC subnets, maintain the subnet bus voltage and ensure the power quality of the $\mathrm{H} \mathrm{MG}[14,15]$. The optimal design of the BIC circuit topology and the corresponding control strategy is the basis of the HMG.

The topology of BIC in HMG can be divided into single-phase and three-phase converter from the phase number. It can be divided into two levels, three levels and multi-levels converter from the electrical level. It can also be divided into single-stage, two-stage cascade, multi-stage cascade converter from the stage number. From whether with the isolation transformer, it can be divided into isolation and Non-isolated converter [16] [17]. In addition, the HMG with higher voltage level adopts the topology of modular multilevel converter and the cost is expensive. At present, BIC mostly adopts the topology of three-phase two-level PWM converter. The circuit structure is simple and easy to implement, but it also has the disadvantages of the insufficient transmission capacity, the small output voltage and the large harmonic current. Therefore, in the HMG system with large capacity demand and high voltage level of DC subnet, three-level topology can be used to replace the traditional three-phase two-level topology for the BIC to improve the transmission capacity and the DC bus voltage level.

The BIC based on the three-level topology has many advantages compared to the two-level topology, but it also has inherent problems of the neutral point potential fluctuation [18]. Furthermore, it will cause the DC bus voltage fluctuation in the AC-DC HMG, which also through the BIC affects the power quality of the AC subnet. Therefore, it is necessary to solve the midpoint potential imbalance of the BIC with three-level topology.

The paper [19] analyzed the causes of the neutral point potential fluctuations and pointed out that the adjustable range of the midpoint potential is positively related to the power factor and negatively related to the degree of modulation. If exceeding this range, the neutral point potential cannot be completely balanced and it is affected by the DC capacitance, the amplitude of phase current, and the power factor. There are many neutral point potential adjustment methods based on space vector pulse width modulation (SVPWM), which can be divided into passive control method, hysteresis control method and active control method [20]. Passive control method is only suitable for complete balance and constant load, so 
it is difficult to apply in the AC-DC HMG. The hysteresis control method and active control method are closed loop control with good robustness, but they are not combine the methods with the control schemes of the BIC.

In this paper, the BIC with the three-level topology is applied to the AC-DC HMG. For the neutral point potential fluctuation caused by the three-level topology, a droop control scheme with midpoint potential hysteresis regulation link is designed. The principle of neutral point fluctuation and the process of hysteresis control are detailed introduced. The detailed design of dual-mode control strategy based on droop curve are also given. Finally, the effectiveness and feasibility of the proposed method are verified by simulation.

\section{The topology of the AC-DC HMG and the BIC}

The topology of the AC-DC HMG this paper is shown in Fig. 1.

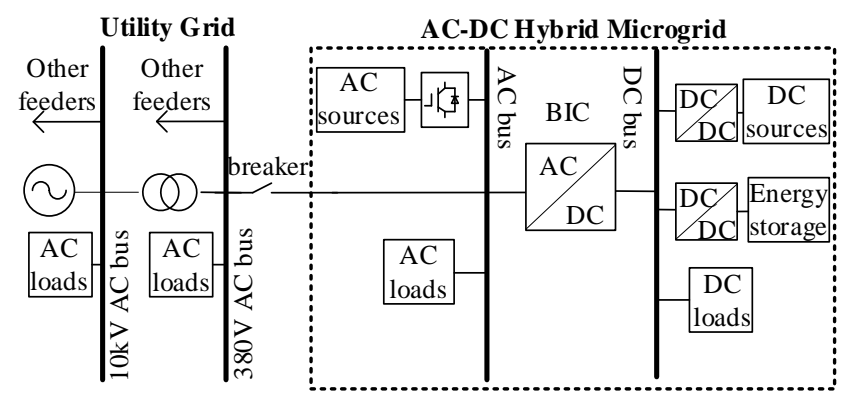

Fig. 1. Topology of the AC-DC HMG.

It consists of AC sources and loads, DC sources and loads, the energy storage (ES) system and the BIC between $\mathrm{AC}$ and $\mathrm{DC}$ buses.

The topology of the BIC in AC-DC HMG is shown in Fig. 2. $u_{\mathrm{sa}}, u_{\mathrm{sb}}$, and $u_{\mathrm{sc}}$ are the phase voltages of the utility grid; $i_{\mathrm{sa}}, i_{\mathrm{sb}}$, and $i_{\mathrm{sc}}$ are the three phase currents of the utility grid; $i_{\mathrm{fa}}, i_{\mathrm{fb}}$, and $i_{\mathrm{fc}}$ are the three-phase output currents of the BIC; $u_{\mathrm{aca}}, u_{\mathrm{acb}}, u_{\mathrm{acc}}$ are three-phase voltages of the AC subnet; $i_{\text {aca }}, i_{\text {acb }}, i_{\text {acc }}$ are three-phase currents of the AC subnet; $i_{\mathrm{O}}$ is the neutral point current on the DC side; $u_{\mathrm{c} 1}, u_{\mathrm{c} 2}$ are the voltages of the two capacitors on the DC side, respectively; $u_{\mathrm{dc}}$ is the voltage of the DC bus.

\section{The proposed control strategy}

\subsection{Analysis of the neutral point potential fluctuation}

The midpoint potential $\Delta u$ refers to the difference between the capacitor voltages $u_{\mathrm{c} 1}$ and $u_{\mathrm{c} 2}$ on the dc side.

$$
\Delta u=u_{c 1}-u_{c 1}
$$

The midpoint current $i_{\mathrm{O}}$ is the current flowing out of the midpoint of the capacitor, that is the difference between the current flowing into the capacitor $\mathrm{C} 1$ and $\mathrm{C} 2$.

$$
i_{o}=i_{c 1}-i_{c 2}
$$

Assuming that the two capacitors on the dc side are equal, $\mathrm{C}=\mathrm{C} 1=\mathrm{C} 2$, the fluctuation of the midpoint potential is completely determined by the midpoint current $i_{\mathrm{O}}$.

$$
\Delta u=u_{c 1}-u_{c 1}=\frac{\int i_{c 1}}{C}-\frac{\int i_{c 2}}{C}=\frac{\int i_{o}}{C}
$$

When the SVPWM method is used for modulation, some switching states will generate a midpoint current that affects the balance of the neutral point potential. Taking the $\mathrm{j}(\mathrm{j}=\mathrm{a}, \mathrm{b}, \mathrm{c})$ phase as an example, its switching state function:

$$
S_{j}=
$$

( $p$ The $j$-phase bridge arm only has $T_{j 1}$ and $T_{j 2}$ turned on $\left\{\right.$ o The $j-$ phase bridge arm only has $T_{j 2}$ and $T_{j 3}$ turned on $n$ The $j$-phase bridge arm only has $T_{j 3}$ and $T_{j 4}$ turned on

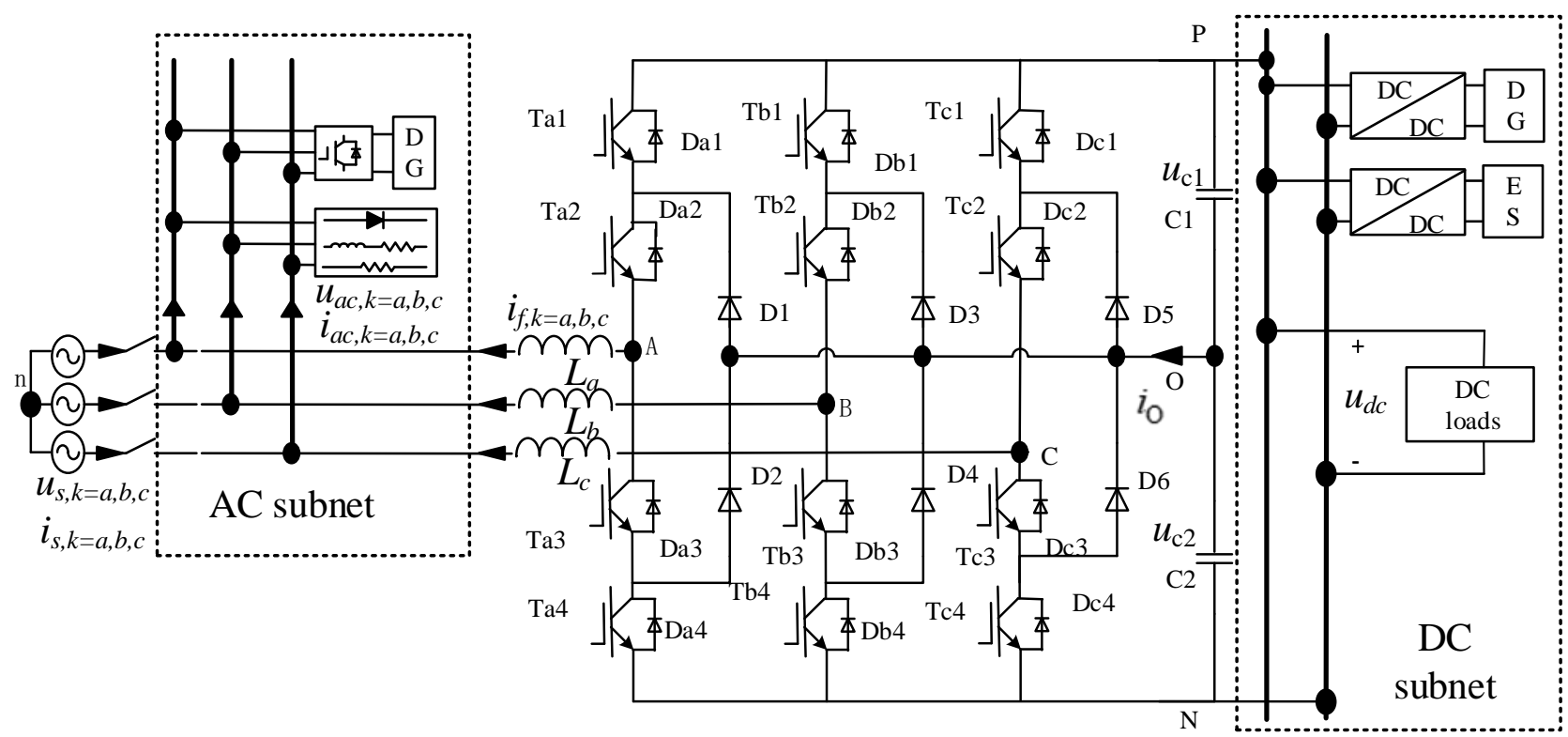

Fig. 2. Topology of the three-level based BIC in the AC-DC HMG. 
Every phase has three switching states: $p, o$, and $n$. The three-phase, three-arm bridge has a total of $3^{3}=27$ combinations of switching states, forming 19 voltage vectors, which can be divided into zero vector, small vector, and medium vectors and big vectors, according to the magnitude of the vector mode as shown in Fig. 3.

Among them, 3 zero vectors and 6 large vectors do not generate midpoint current and do not affect the midpoint potential. 12 small vectors compose 6 pairs of redundant small vectors can generate forward direction midpoint current and each pair of redundant small vectors generate the corresponding opposite direction neutral point current. Therefore, the opposite effect can ensure the stability of the neutral point potential. The corresponding relationship is shown in Table I. Six medium vectors which produce midpoint current also affect the neutral point potential. As the DC bus voltage deteriorates, the power quality of the AC-DC HMG will decrease.

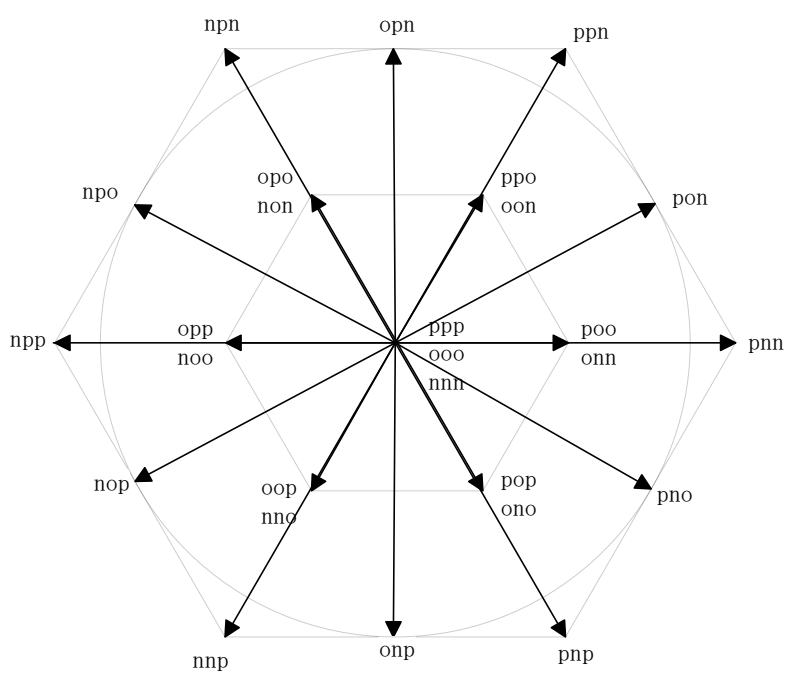

Fig. 3. Space voltage vectors with their switching states for three-level.

Table 1. Positive and negative vector corresponding to the midpoint current

\begin{tabular}{cccc}
$\begin{array}{c}\text { positive small } \\
\text { vectors }\end{array}$ & $\begin{array}{c}\text { neutral point } \\
\text { current } \boldsymbol{i}_{\boldsymbol{O}}\end{array}$ & $\begin{array}{c}\text { negative small } \\
\text { vectors }\end{array}$ & $\begin{array}{c}\text { neutral point } \\
\text { current } \boldsymbol{i}_{\boldsymbol{O}}\end{array}$ \\
\hline onn & $i_{f a}$ & poo & $-i_{f a}$ \\
\hline ppo & $i_{f c}$ & oon & $-i_{f c}$ \\
\hline non & $i_{f b}$ & opo & $-i_{f b}$ \\
\hline opp & $i_{f a}$ & noo & $-i_{f a}$ \\
\hline nno & $i_{f c}$ & oop & $-i_{f c}$ \\
\hline pop & $i_{f b}$ & ono & $-i_{f b}$ \\
\hline \hline
\end{tabular}

\subsection{Neutral point potential hysteresis regulation}

The basic principle of the Seven-segment control mode in SVPWM modulation is shown in Fig. 4.

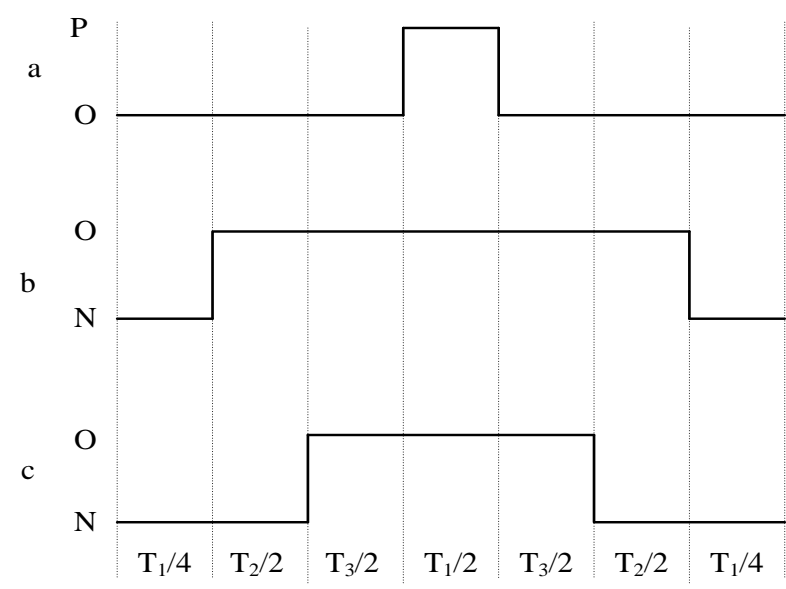

Fig. 4. Seven-segment SVPWM modulation.

If the action time of positive and negative small vectors are equal in a period, the integral value of the current flowing into the midpoint of the capacitor is zero, which will not affect the midpoint potential. If the medium vector is used in a certain period, the integral value of the neutral point current must not be zero, which will cause the change of the midpoint potential. Therefore, it is necessary to adjust the action time of the positive and negative small vectors to make the midpoint current generated offset the neutral point potential.

The basic principle of the hysteresis regulation of the neutral point potential is to set the hysteresis adjustment range and calculate the adjustment effect of each small vector on the neutral point potential. When the neutral point potential exceeds this range, the small vector with midpoint potential balance capability is used to adjust the neutral point potential. The specific adjustment steps are as follows:

1) From the corresponding relationship shown in Table 1, the neutral point current $i_{\mathrm{O}}$ depending on the small vector and the BIC output current if determines the adjustment effect of each small vector on the neutral point potential. Each pair of redundant small vectors corresponding to opposite direction neutral point current has opposite regulatory effect.

2) Set the neutral point potential hysteresis interval $m$ $=\left(-u_{\mathrm{d}}, u_{\mathrm{d}}\right)$, detect the neutral point potential $u_{\mathrm{O}}$ and its deviation degree, When the neutral point potential is within $\mathrm{m}$, maintain the original small vector time.

3) When the neutral point potential exceeds the interval $\mathrm{m}$, adjust the neutral point potential by increasing the small vector time with neutral point potential equalization capability. Adjusting the size of the hysteresis zone $\mathrm{m}$ can control the fluctuation range of the neutral point potential.

\subsection{The overall BIC control strategy}

The paper considers the AC-DC HMG in the low voltage environment, which is at the end of the power system, and its line impedance $\mathrm{R}>>\mathrm{L}$. Therefore, the uac-Pac and udc-Pdc droop control strategy is selected to control the $\mathrm{BIC}$ under grid-connected and island mode:

In Fig. 5, the AC-DC HMG operates in the grid-connected mode, due to the support of the utility 
grid, the amplitude and the frequency of the $\mathrm{AC}$ bus voltage will not change, the BIC can absorb or supplement redundant energy with the help of the utility grid. The DC bus voltage can be controlled by the constant voltage scheme and the flow power of BIC is determined by the load condition of the AC and DC subnet. The AC-DC HMG operates in the island mode, due to the lack of the utility grid, the voltage quality of $\mathrm{AC}$ and DC subnet decreases. The BIC needs bidirectional power transmission, which based on the preset droop curve, to ensure the power balance and voltage stability of the AC and DC subnet.

The proposed droop control strategy with the midpoint potential hysteresis regulation link of the BIC is shown in Fig. 6 and the proposed control strategy is implemented as follows:

1) Detect the AC and DC bus voltage, then generate the reference value $P_{\text {tref }}$ of the transmitted active power through the droop or constant voltage regulator (VR).

2) Generate the reference value $Q_{\text {tref }}$ of transmitted reactive power through the frequency closed loop control.

3) Extract harmonic components from the current of AC subnet to generate reference current values of $i_{\mathrm{fd} 1}$ and $i_{\text {fq1 }}$ and get the transmission active and reactive reference current value $i_{\mathrm{fd} 2}$ and $i_{\mathrm{fq} 2}$ from $P_{\text {tref }}$ and $Q_{\text {tref }}$, and then superimpose the reference current to generate the final reference current $i_{\text {fdref }}$ and $i_{\text {fqref }}$.

4) Pass the difference value between the final reference current and the output current of the BIC through the current regulator (CR) and the decoupling link to generate the modulation wave in the dq0-frame, convert it to the $\alpha \beta$-frame and enter the SVPWM link with $u_{\mathrm{dc}}$.

5) Apply midpoint current hysteresis regulation to optimize the SVPWM link and generate PWM signal to drive the BIC works.

It is worth noting that the proportional integral controller is selected as VR to realize the zero difference adjustment of the bus voltage and the quasi proportional resonance controller is selected as $\mathrm{CR}$ to guarantee the accurate and fast tracking of the fundamental and harmonic currents.

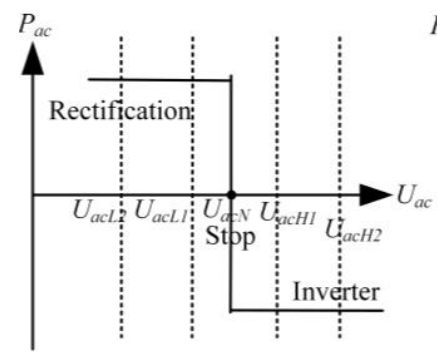

(a)

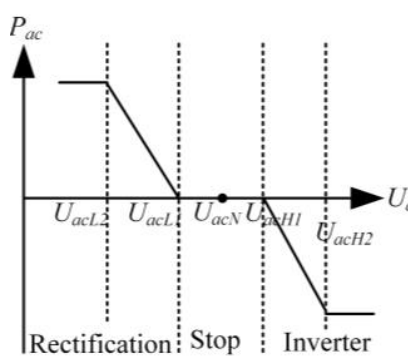

(c)

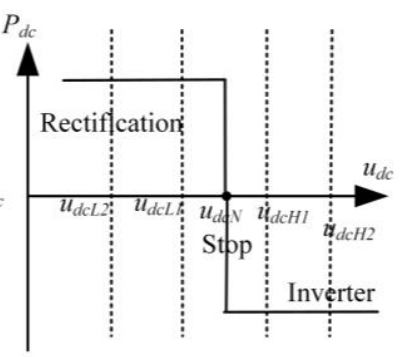

(b)

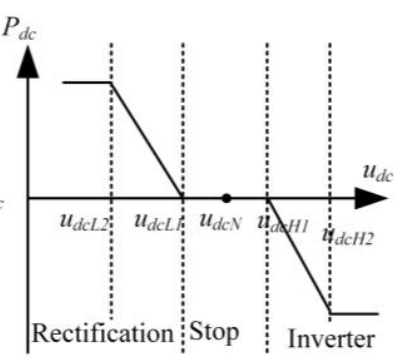

(d)
Fig. 5. The droop curve of the BIC. (a) the relationship between Uac and Pac in grid-connected mode. (b) the relationship between udc and Pdc in grid-connected mode. (c) the relationship between Uac and Pac in island mode. (d) the relationship between udc and Pdc in island mode.

\section{Simulation results}

In order to verify the effectiveness of the proposed control strategy of BIC, a simulation model of the AC-DC HMG was built in MATALB/Simulink as shown in Fig. 2. The specific simulation parameters are shown in Table 2.

The process of the neutral point potential hysteresis regulation is shown in Fig. 7 and simulation waveform of the midpoint potential is shown in Fig.8.

In Fig. 7, the small vector pair used in the synthesis vector was judged from the region of the reference

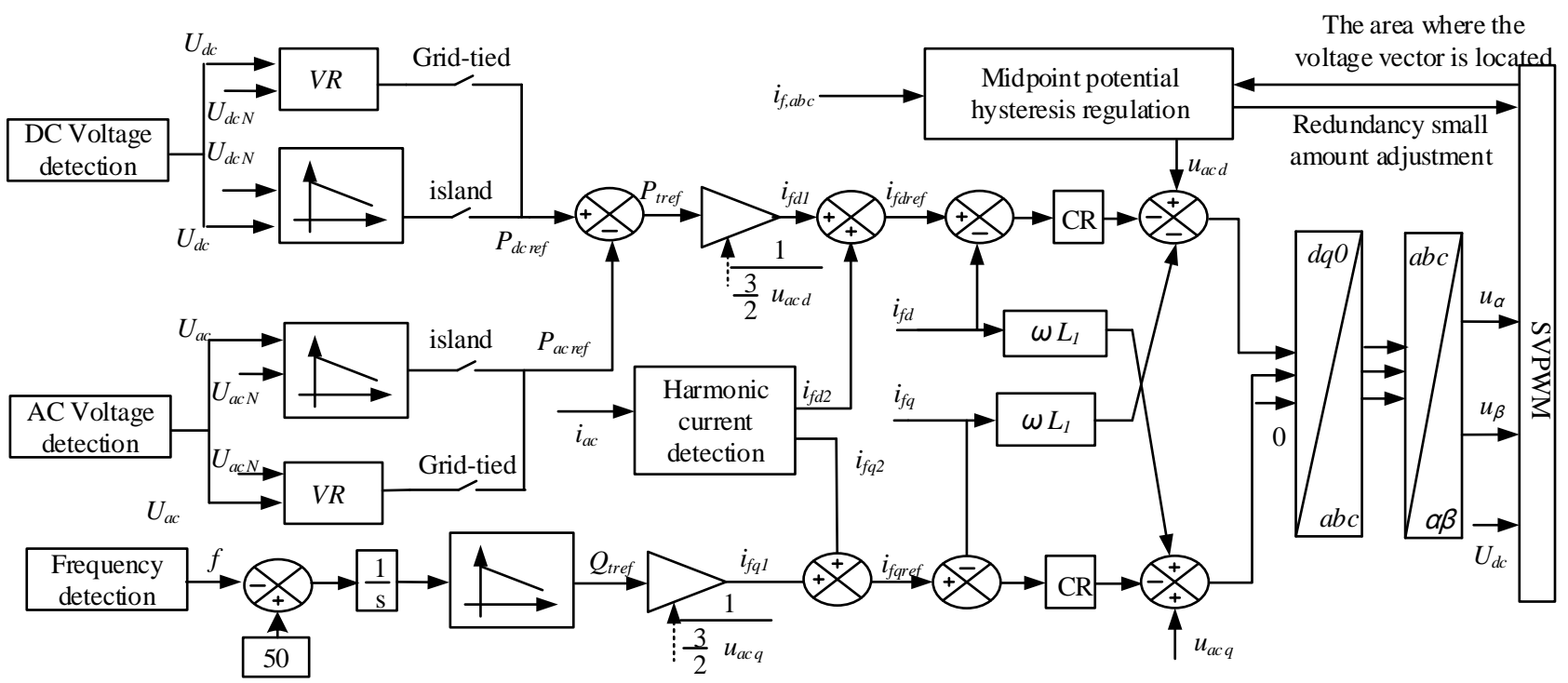

Fig. 6. The proposed droop control strategy with the midpoint potential hysteresis regulation link of the BIC. 
voltage, and then calculated the midpoint current obtained from the BIC output current. If the midpoint current is too small, the regulating effect of midpoint action time on the midpoint potential is limited, Therefore, when the absolute value of the neutral point current is greater than $i_{\mathrm{k}}$ and the midpoint potential exceeds the hysteresis range $m=\left(-u_{\mathrm{k}}, u_{\mathrm{k}}\right)$, the action time of the small vector was adjusted.

It can be seen in Fig. 8 that the use of if at 1.0s for the hysteresis adjustment of the midpoint potential, the hysteresis interval $\mathrm{m}=(-1 \mathrm{~V}, 1 \mathrm{~V})$ and the midpoint potential is limited to the hysteresis interval $\mathrm{m}$.

Table 2. System Parameters in the Simulation and Experiment

\begin{tabular}{lll}
\hline \hline & Parameters & Simulation \\
\hline \multirow{2}{*}{$\begin{array}{l}\text { Utility } \\
\text { Grid }\end{array}$} & nominal voltage $u_{\mathrm{s}}$ & $380 \mathrm{~V} / 50 \mathrm{~Hz}$ \\
& line inductance & $0.3 \mathrm{mH}$ \\
& line resistance & $0.1 \mathrm{ohms}$ \\
\hline \multirow{2}{*}{ AC-DC } & AC-link voltage $u_{\mathrm{ac}}$ & $380 \mathrm{~V} / 50 \mathrm{~Hz}$ \\
$\mathrm{HMG}$ & AC-link voltage $u_{\mathrm{dc}}$ & $750 \mathrm{~V}$ \\
& & $6 \mathrm{mH}$ \\
& L-LPF $\mathrm{L}_{\mathrm{a}}, \mathrm{L}_{\mathrm{b}}, \mathrm{L}_{\mathrm{c}}$ & $10 \mathrm{kHz}$ \\
BIC & switch frequency & $2000 \mu \mathrm{f}$ \\
& DC-link capacitor $\mathrm{C}_{1}$ & $2000 \mu \mathrm{f}$
\end{tabular}

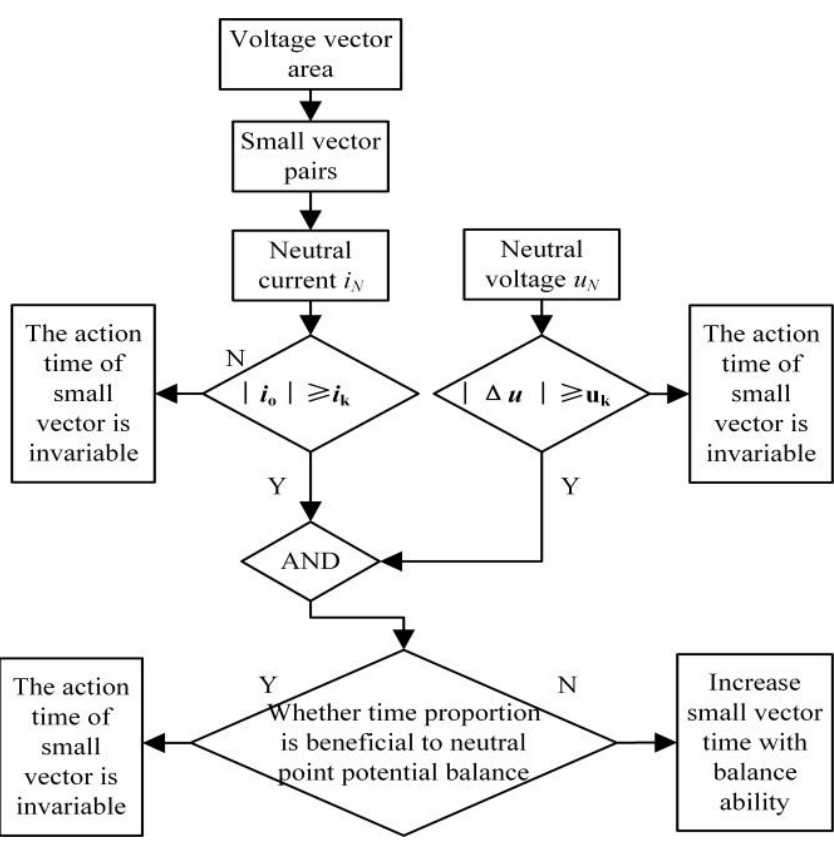

Fig. 7. Neutral point potential hysteresis loop adjustment.

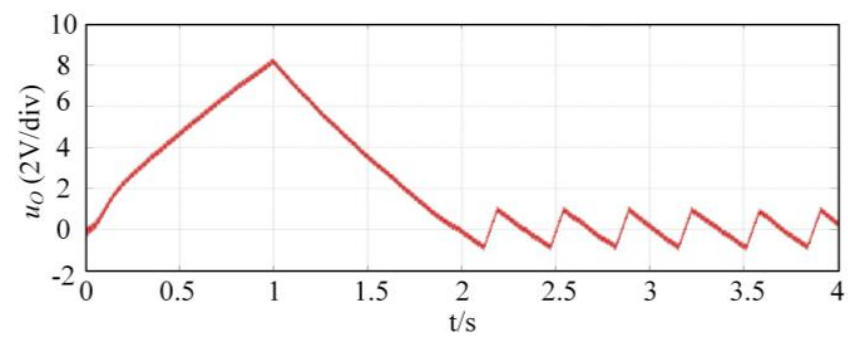

Fig. 8. Midpoint potential
The simulation waveform of DC bus voltage in the AC-DC HMG under the grid-connected mode as shown in Fig. 9. From Fig. 9, the load fluctuation was simulated at $0.2 \mathrm{~s}$ and $0.4 \mathrm{~s}$ respectively, and it can be seen that the DC voltage can be restored to the initial state within $0.2 \mathrm{~s}$.

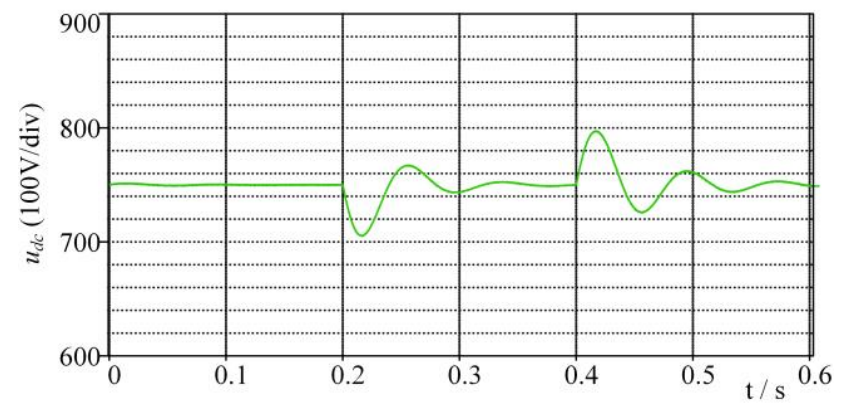

Fig. 9. Simulation waveform of the DC bus voltage.
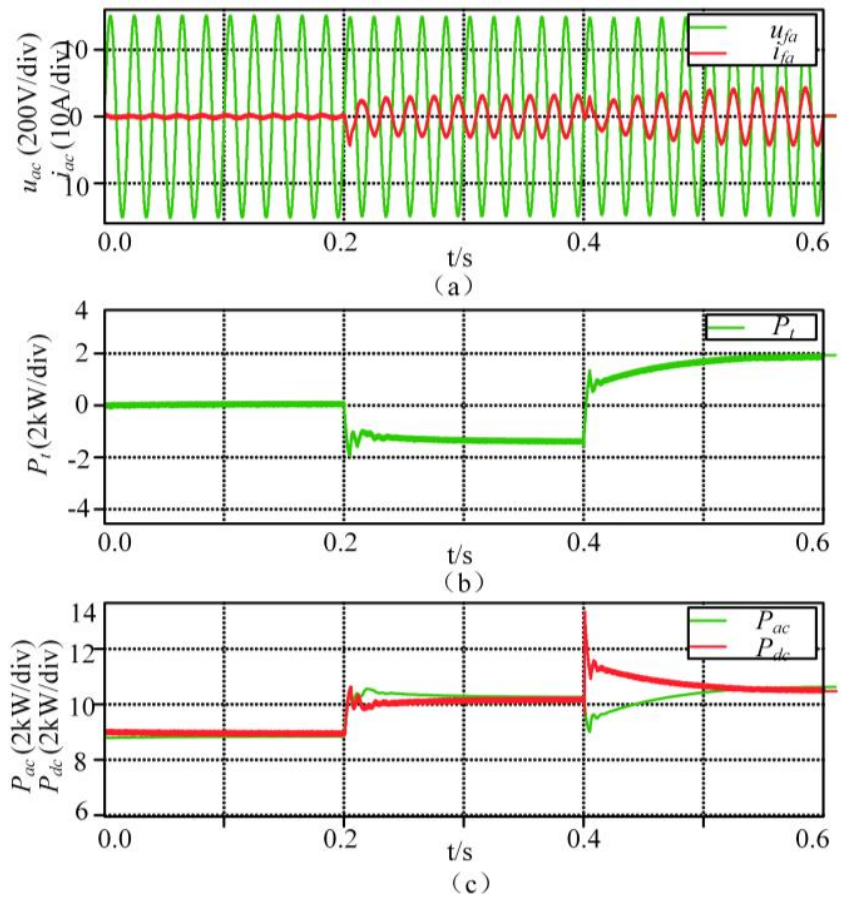

Fig. 10. Simulation operation of the BIC in the island mode. (a) the BIC phase-A output voltage and current. (b) the BIC output active power. (c) the output active power of the AC and DC subnet.

Fig. 10 shows the power transmission of the BIC during the island operation. The phase-A output voltage and current of the BIC, the output active power of the BIC and the output active power of the AC and DC subnet were respectively shown in Fig. 10(a), (b) and (c). It can be seen that the BIC operates in the shutdown mode at $0 \sim 0.2 \mathrm{~s}$. When the load fluctuated between $0.2 \mathrm{~s}$ and $0.4 \mathrm{~s}$, the BIC switch to the inverter mode and the rectifier mode respectively to transmit the corresponding active power.

From Fig. 8 to 10 , it can be seen that the designed midpoint potential regulation method can effectively suppress the voltage fluctuation of the midpoint, at the same time, it will not affect the voltage and power control target of the BIC under the grid-connected and island mode. 


\section{Conclusion}

Aiming at the disadvantages of the BIC with the two-level PWM topology in the AC-DC HMG, this paper proposes to apply the three-level topology based BIC to the system, which requires the large transmission power and the high voltage level. In addition, an improved control strategy based on droop control principle and midpoint hysteresis regulation has been designed. The proposed control scheme has superior performance in both grid-connected and island mode as:

1) The fluctuation of neutral point voltage was suppressed by the additional neutral point potential hysteresis regulation, which can guarantee the voltage quality of the DC subnet.

2) The additional neutral point potential regulation link does not affect the realization of other control objectives of the BIC in both grid-connected and island mode.

The authors would like to express gratitude to the major science and technology projects of Shanxi Province (20181102028) and Shanxi Science and technology project (520530180011).

\section{References}

1. P. Wang, X. Liu, Jin C, P. C. Loh and C. Jin, 2011 IEEE Power and Energy Society General Meeting, 1-8(2011)

2. C. Wang, X. Li, L. Guo and Y. L. Lei, TPE, 29, 6162-6177(2014)

3. D. I. Döring, D. Dhua, S.J. Huang, and Q.W. Wu, IJEEET, 7, 165-171(2018)

4. R. Baharom, M. N. Seroji, and A. I. M. Yassin, IJEEET, 7, 159-164(2018)
5. J. Joseph and A. Kumar M K, IJEEET, 1, 209-213(2015)

6. N. Hatziargyriou, H. Asano, R. Iravani and C. Marnay, PEM, 78-94(2007)

7. T. R. Oliveira, W. W. A Goncalves and P. F. Donoso-Garcia, TSG, 8, 2597-2607(2017)

8. A. Sallam, M. E. Nassar, R. A. R. Hamdy and M. M. A. Salama, 2017 IEEE Electrical Power and Energy Conference, 1-5(2017)

9. A. Lachichi, A. Junyent-Fete and T. Green, IECON 2018. 44th Annual Confefence of the IEEE Industrial Electronics Society, 3973-3978(2018)

10. N. Eghtedarpour, E. Farjah, TSG, 5, 1494-1505(2014)

11. M. Shahparasti, M. Mohamadian, P. T. Baboli, and A Yazdianp, TSG, 1-11(2015)

12. X. Chen, G. Zhang, Power Electronics\&Motion Control Conference, 22-26(2016)

13. X. Liu, P. Wang and P. C. Loh, TSG, 2 , 278-286(2011)

14. F. Nejabatkhah, Y. W. Li, TPE, 30, 7072-7089(2015)

15. M. Khederzadeh, M. Sadeghi, IET GTD, 10, 3539-3546(2016)

16. H. S. Ribeiro, B. V. Borges, TIE, 61, 2264-2277(2014)

17. J. Zang, M. M. Jovanovic and F. C. Lee, APEC 99 Fourteenth Annual, 335-341(1999)

18. W. Song, G. Chen, X. Ding, M. Shu, TCES, 3, $1-6(2006)$

19. N. Celanovic, D. Boroyevich, TPE, 15, 242-249(2002)

20. W. Yao, Z. Lv, W. Fei, PCSEE, 25, 92-96(2005) 